\title{
Meditation music improved the quality of suturing in an experimental bypass procedure
}

\section{Muhammad, Sajjad}

2019-08

Muhammad, S, Lehecka, M , Huhtakangas , J, Jahromi , B R, Niemelä , M \& Hafez , A 2019 , ' Meditation music improved the quality of suturing in an experimental bypass procedure ' , Acta Neurochirurgica , vol. 161 , no. 8 , pp. 1515-1521 . https://doi.org/10.1007/s00701-019-03976-4

http://hdl.handle.net/10138/325824

https://doi.org/10.1007/s00701-019-03976-4

unspecified

acceptedVersion

Downloaded from Helda, University of Helsinki institutional repository.

This is an electronic reprint of the original article.

This reprint may differ from the original in pagination and typographic detail.

Please cite the original version. 
1 Meditation music improved the quality of suturing in experimental bypass procedure

2 Sajjad Muhammad MD, $\mathrm{PhD}^{1}$, Martin Lehecka MD, PhD ${ }^{1}$, Justiina Huhtakangas MD, PhD ${ }^{1}$, Behnam

3 Rezai Jahromi, $\mathrm{MD}^{1}$, Mika Niemelä, MD, $\mathrm{PhD}^{1}$, Ahmad Hafez MD, $\mathrm{PhD}^{1 *}$

Affiliations of Authors

$6{ }^{1}$ Department of Neurosurgery, University of Helsinki and Helsinki University Hospital, Helsinki,

$7 \quad$ Finland

8 *Correspondence:

9 Ahmad Hafez, MD, PhD

10 University of Helsinki and Helsinki University Hospital

11 Department of Neurosurgery

Topeliuksenkatu 5

P.O. Box 266, Fin-00029-HUS,

Helsinki, Finland.

Email: ext-ahmad.hafez@hus.fi/ahmadhafez1965@gmail.com

Tel: 00358-405885513

Acknowledgments:

- We are thankful to Ehrnrooth Foundation for the Funding to first author (S M) for a clinical vascular and skull base fellowship, and for awarding the last author (A H) the C. Ehrnrooth Fellowship (Fondation de.Luxembourg) at the department of Neurosurgery in Helsinki.

- This research did not receive any specific grant from funding agencies in the public, commercial, or not-for-profit sectors. 
ABSTRACT

BACKGROUND: Neurosurgeons are vulnerable to additional noise in their natural operating environment. Noise exposure is associated with reduced cognitive function, inability to concentrate, and nervousness. Mediation music provides opportunity to create a calmer environments which May reduce stress during surgery.

METHODS: A pilot study was performed to find suitable task, meditation music of surgeon's choice, operation noise and to reach a certain level of training. For the main experiment, two neurosurgeons with different microsurgical experience used real operation noise and meditation music with delta waves as mediating music. Each surgeon performed 10 training bypasses ( 5 with noise and 5 with music) with 16 stitches in each bypass. The total time to complete 16 stitches, number of unachieved movements (N.U.Ms), length of thread consumed and distribution of the stitches were quantified from the recorded videos and compared in both groups.

RESULTS: The N.U.Ms were significantly reduced from $109 \pm 38$ with operation room (OR)noise to $38 \pm 13$ ( $\mathrm{p}<0.05$ ) with meditating music in novice surgeon. Similar results were found if the experienced surgeon performed the same task [(from $29 \pm 6.94$ to $14 \pm 3.36(\mathrm{p}<0.05)]$. The total time utilized for the sixteen stitches was slightly improved (not significantly) in novice surgeon and unchanged in experienced surgeon. However, the thread length used for 16 stitches was significantly different with OR-noise in comparison to meditating music in both surgeons. The distribution stitches showed a non-significant trend towards a uniform distribution with meditation music in both surgeons.

CONCLUSIONS: Meditation music of surgeon's choice is a simple method that improved quality of bypass suturing in experimental bypass procedure.

Key words: Operation Room, Noise, Meditation, Music, Neurovascular, Bypass

\section{Introduction}

Neurosurgery includes many highly skill-demanding and stressful procedures, such as bypass surgery. The "stress rate" and drivers of stress are different for each neurosurgical procedure and for each neurosurgeon. Stitching during bypass surgery is one of the procedures, which holds high level 
of stress. It necessitates a focused attention to achieve good quality in optimal time. The goal of high quality stitching in a short period of time can lead to stress and anxiety[11].

Environment, including the noise, in the operation room is an important factor specially in distracting surgeon's concentration and increasing the stress [14]. Stress is known to influence the heart beat variability and other vegetative function [33]. Hence, the strategies to reduce distraction, anxiety, and release the stress of the surgeon may be helpful to improve the quality of surgery

Meditating music may help to circumvent debilitating effect of stress on the fine motor skills of the neurosurgeon. Meditation and meditating music has been shown to relieve stress, and improve learning $[1,26]$. There is increasing evidence that music attenuates symptoms in a variety of diseases including Parkinson's disease, dementia and attention deficit syndrome [23]. Intense pleasure in response to music activates the dopaminergic system in striatum and mesolimbic system. Dopamine release in the central nervous system has implication in combating stress [28, 29]. The dopaminergic and serotoninergic deep nuclei are linked to the motor cortex supporting the possibility that fine motor skills might be influenced by the meditating music. Evidence from literature supports the notion that sound, the auditory system and the motor system are interconnected and hence may control the fine motor movements [10, 37].

We aim to investigate the influence of meditating music and compared it with the influence of operation room noise on surgical performance. We introduced meditating music on our already established experimental bypass surgery to investigate the difference in quality of suturing during OR-noise and meditation music of surgeon's choice.

\section{Materials and methods}

We recorded the videos of stitching artificial vessels and investigated different parameters to quantify the quality of bypass suturing. These parameters included unachieved movements of surgeon, distribution of the stitches, time utilized and total length of thread used for sixteen microsurgical stitches. These parameters were evaluated in an environment with routine operation room noise in comparison to meditating music. We investigated the above-mentioned parameters in two surgeons with different training experience. The experiment was conducted at the Neurosurgical Department in the Helsinki University Hospital, using an OPMI PENTERO 900 ZEISS inside an OR (Fig.1A)

In this experimental study, all training tasks simulating bypass suturing were end-to-side procedures. The tasks were performed by first author (S.M), a fully training EU board certified neurosurgeon 
(2017) worked as a clinical fellow in neurovascular and skull base microneurosurgery for one year (2018), and the last author (A.H), who finished his residency 20 years ago and who has performed 1,500 different experimental bypass procedures over past 4 years, between June 2014 and July 2017, in the Department of Neurosurgery, Helsinki University Hospital, Helsinki, Finland.

(1)

(1)

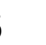

\section{Experiment Design}

A vessel wall suturing procedure was simulated. The two participants were asked to suture an already cut in scaphoid-shaped silicon tube. They were required to distribute 16 stitches with four knots.

We designed our model by developing a shape similar to the shape of bypass suturing end-side interrupted stitching. The shape was developed, using a millimeter paper (Fig. 1B). We chose the $4 \mathrm{~mm}$ diameter tube and 16 stitches to be used with 4 corner stations, which allow measuring the distances between stitches, which perfectly supposed to be one millimeter (Fig. 1C).

The participants were asked to suture in the same order; the numbers ( 1 to 4 ) in the model were used to keep participants direction and order of suturing (Fig. 1B). The magnification during suturing was (8 times) in all 20 cases.

We used same 9-0 suturing material (PROLENE, $13 \mathrm{~cm}, 5.0 \mathrm{~mm}, 3 / 8 \mathrm{c}$, ETHICON) and same micro-instruments (i.e. scissors, needle holder and forceps) in all procedures (Fig 1A).

All videos were analyzed blindly after finishing all the cases. Suturing time was measured from video. The length of thread consumed by the procedures was measured by measuring the length of remaining thread. The repeated movements, which did not achieve the intention goal (e.g. catching the needle, catching the tube wall with tweezers) were counted carefully from videos. The distribution of stitches was quantified by giving a score based on the uniformity of distribution (distance between each stitch) of 16 stitches on the vessel wall (Figure 1C).

\section{Statistical analysis:}


The data were analyzed using GraphPad Prism 5.00 (GraphPad Software, San Diego, CA, USA). After testing for normality, unpaired t test and welch, s correction was performed in case of nonnormal distribution to compare two groups.

Data is expressed as mean \pm SEM, a p value $<0.05$ was considered as significant difference.

\section{Results:}

Results of video analysis of the procedures for novice surgeon and experienced surgeon using original operation noise and meditating music are summarized in table 1. Interesting, the N.U.Ms were significantly reduced from 109. \pm 38 with OR-noise to $38 \pm 13(\mathrm{p}<0.05)$ with meditating music in novice surgeon (Fig. 2 B). Similar results were found if the experienced surgeon performed the same task. The N.U.Ms was reduced from $29 \pm 7$ to $14 \pm 3(p<0.05)$ showing that meditation influenced positively the surgeons motor skills independent of experience and training (Fig.2 A \&B). Stitch distribution did not differ significantly (Fig 5 A \& B).

Moreover, the length of thread used for the sixteen stitched was significantly reduced from $8 \pm 1 \mathrm{~cm}$ with OR-noise to $5 \pm 0.46$ ( $\mathrm{p}<0.05$ ) with meditating music in novice surgeon (Fig. 4B). Similar results with significantly less use of thread for 16 stitches was quantified in experienced surgeon (Fig. 4A). showing a reduced length of thread needed for 16 stitched from $9 \pm 0.42 \mathrm{~cm}$ to $7 \pm 0.45 \mathrm{~cm}(\mathrm{p}<0.05)$ (Fig. 4 A \& B). The total time utilized for the sixteen stitches was slightly improved (not significantly) in novice surgeon (Fig. 3B) and unchanged in experienced surgeon (Fig. 3A). Interestingly, the N.U.Ms fluctuated during the OR-noise and they were less fluctuating with meditating music over the time of five repeated experiments (table 1).

\section{Discussion}

This study assessed the influence of meditating music on the performance of the neurosurgeons, recruiting the highly demanding procedure: the bypass.

Meditation may reduce the stress and influence the motor skills of surgeons. However, music and task is a complex field and may depend on personality of surgeon, choice of music, and other many other factors. Up to our knowledge this is the first study showing improvement of the quality of stitching with music in an experimental setting. 
146 Previous studies showed the intervention of external distraction on the neurosurgical performance

$147[13,14]$. Noise is a known health hazard leading to stress, and decrease the concentration [15, 30].

148 Relaxing atmosphere of the operation room might be a factor, which helps surgeon to achieve better performance.

150 The meditation has been shown in various studies to influence different brain areas $[5,9,33,35]$. It 151 has been used to reduce the stress, anxiety and depression in patients and in healthy individuals [21, 152 31]. Music is known to have influence on the mood and physiological responses [2, 29]. It has been used as an independent nursing intervention for preoperatively in patients having day surgery [7].

154 155 156 157 158 159 160 161 162 163 164 165 166 167 168 169 170

The trainees experience a different levels of stress and anxiety before and during surgical procedures $[3,12,22]$. Neurosurgical operations hold high level of anxiety, demands certain level of hand-eye coordination, and fine manipulation $[4,6,8,17,36]$.

The senior and expert neurosurgeons compete better with complex surgeries and stressful situation than novice ones $[5,16]$. In contrast, at the time of new resident the operation room noise and movement levels are changed dramatically. Opposite to the norm and trend, in which the seniors get more calm atmosphere, the novice and less experience neurosurgeons in need of more comfortable and less noisy operation room[14].

Meditation and meditating music is becoming increasingly popular and achieving scientific support [20]. It could help in stressful circumstances and overcoming part of the anxiety associated with surgical procedures. Our study showed that meditating music improved the quality of bypass independent of training level. Fortunately, not all neurosurgical procedures hold such high level of challenge with associated anxiety and stress. Hence, the result could be less dominant in simple routine and daily procedures.

The comfort and relaxed hearing voice (meditating music) may improve efficiency and decrease the operative time. Our results showed improved efficiency measured by number of unachieved movements during surgery (Fig.2 A \& B). However, our result showed only a trend toward improved time (Fig 3 A \& B). The comfortable voice and experience of the surgeon are directly related, and the lack of experience would translate directly to slower, more hesitating movement, and less flexibility. In our experimental task the less experience neurosurgeons saved more length of the thread but consumed extra time and repeated more unachieved movements (Table 1).

Meditating music is well established as a way of adjusting the animal and human behaviors [19, 32, 34]. However, different meditation forms could affect different cognitive processes [20]. Using 
meditating music could calm body movements and optimizes movements, which help in achieving better motor performance [24].

Music acts as non-pharmacological intervention that attenuates a variety of symptoms in various diseases, such as Parkinson's disease, atrial hypertension, dementia, and attention- deficit/ hyperactivity disorder [23]. It changes the concentrations of dopamine, serotonin in deep brain nuclei linked to the motor area [37]. The relationship among sound, auditory circuity, and motor systems which control the fine movements is well established in the literature [37]. The hearing stimuli has a remarkable ability to drive rhythmic, metrically organized motor behavior [25, 27].

There are inevitable background noise in operation room as well as distractions [18]. Our older study proved the difficulty of engaging in a secondary task during surgery and the negative impact of the voice distraction [14]. The experienced neurosurgeons could compensate the stress of operation atmosphere including the noise. However, the type and the level of distraction in one hand, and the level of the complexity of the procedure in other hand cannot be ignored. When the procedure is high -skill demanding, such as bypass neurosurgery, the external stimulation becomes crucial.

Our data with some limitations show that performance was worse during practicing bypass under the influence of operation room noise compared to the meditating music. The effect was different between the two subjects (neurosurgeons), based on the past experience with the procedure. Even with extra attention paid by neurosurgeons to complete the task, trying ignoring the sound of the operation room, still the irritating sound reflected the performance as well as laid its effect on the results. The price of the noise and the benefit from the meditating music reflected on the number of unnecessary movements before achieving the intentional goal (Fig2 A \& B). For example; penetrating the wall of the tube, tight the stitch, holding the needle, and others.

Finally, even with small number of procedures, learning curve and adaptation with the noisy operating sounds were noticed in our trial. The individual endurance level of tension and ability to cope with noise distraction stands behind differences in handling such matter in our field.

\section{Limitations}

The main limitation is that this study includes a limited number of surgeons; only two surgeons involved, and no randomization of the exercises. Therefore, the results might partially reflects their personal traits and the different volume of bypass training they underwent previously. Moreover, repeating the task and learning effect (learning curve theory) could influence the result. 
Although it is attractive study with important findings, it is less realistic than naturalistic observation. Although suturing style and designed task are recruited from bypass procedure, this task does not simulate all the bypass steps. This study covers a narrow corner in everyday neurosurgical practice. However, it is part of a series of studies focusing on the training quality in neurosurgery.

\section{Conclusions}

We conclude that the noise influenced performance of the surgeon, especially when performing complex task such as bypass procedure. The meditating music could positively impact the automaticity of surgical performance. Level of noise and experience of the neurosurgeons can affect the outcome of the surgery. To achieve better outcome, the noise level inside the operation room and use of individual meditating music may be considered

Table 1. Video analysis of different factors to quantify the quality of bypass procedure for (Novice surgeon), (experienced surgeon), using recording of real operation noise and meditating music

\section{Figure legends}

Figure 1A. Experimental setup and the surgeon and microscope inside the OR. Video recording of bypass suturing, designed task, and silicon tube and suturing instruments are seen on the screen.

Figure 1B. Schematic view of the suturing task. The diamond-shaped "rhombus" with $4 \mathrm{~mm}$ length of its four sides (red arrow). The tube diameter also is $4 \mathrm{~mm}$. The axis located in this direction. The numbers $(1,2,3$, and 4$)$ was sutured first in order and then starting from number 1 clockwise (black curved arrow) to complete the 16 stitches at the end. The background of the design is a millimeter paper was used after suturing to analysis the result.

Figure 1C. Suturing sample captured from a recorded video, after completion of experiment we used the millimeter paper to examine the distribution of the stitches.

Figure 2. Number of unachieved movements with operation noise and after meditating music in both; experienced surgeon $(A), n=5$, unpaired t-test $(p=0.0027)$ and novice surgeon $(B), n=5$, unpaired $t$ test $(\mathrm{p}=0.017)$ 
237 Figure 3. Time of suturing with operation noise and after meditating music in experienced surgeon 238 (A), $n=5$, unpaired t-test $(\mathrm{p}=0.52)$ and novice surgeon $(\mathrm{B}), \mathrm{n}=5$, unpaired t-test $(\mathrm{p}=0.27)$

239 Figure 4. Length of thread used with operation noise and after meditating music in experienced 240 surgeon $(A), n=5$, unpaired t-test $(\mathrm{p}=0.0001)$ and novice surgeon $(\mathrm{B}), \mathrm{n}=5$, unpaired $\mathrm{t}$-test $(\mathrm{p}=0.017)$

241 Figure 5. Distribution of stitches with operation noise and after meditating music in experienced surgeon $(A), n=5$, unpaired t-test $(p=0.073)$ and novice surgeon $(B), n=5$, unpaired t-test $(p=0.57)$

Conflict of interest: All authors certify that they have no affiliations with or involvement in any organization or entity with any financial interest, or non-financial interest in the subject matter or materials discussed in this manuscript.

\section{Compliance with the ethical standard}

Conflict of interest: All authors certify that they have no affiliations with or involvement in any organization or entity with any financial interest, or non-financial interest in the subject matter or materials discussed in this manuscript.

- Funding-No funding was received for this research.

- We are thankful to Ehrnrooth Foundation for the Funding to first author (S M) for a clinical vascular and skull base fellowship, and for awarding the last author (A H) the C. Ehrnrooth Fellowship (Fondation de.Luxembourg) at the department of Neurosurgery in Helsinki.

- Author (S M) declares that he has no conflict of interest. Author (A H) declares that he

- All other authors declare that they have no conflict of interest.

- Ethical approval: This article does not contain any studies with human participants or animals 

performance. Behavioural brain research 256:257-260

2702 2. Artemiou E, Gilbert GE, Sithole F, Koster LS (2017) The Effects of Music during a Physical 271 Examination Skills Practice: A Pilot Study. Veterinary sciences 4 3. Barbosa P, Raymond G, Zlotnick C, Wilk J, Toomey R, 3rd, Mitchell J, 3rd (2013) Mindfulnessbased stress reduction training is associated with greater empathy and reduced anxiety for graduate healthcare students. Education for health 26:9-14 4. Botelho RV, Jardim Miranda BC, Nishikuni K, Waisberg J (2018) Life Expectancy of Brazilian Neurosurgeons. World neurosurgery 114:e857-e860

5. Brefczynski-Lewis JA, Lutz A, Schaefer HS, Levinson DB, Davidson RJ (2007) Neural correlates of attentional expertise in long-term meditation practitioners. Proceedings of the National Academy of Sciences of the United States of America 104:11483-11488

6. Byvaltsev VA, Akshulakov SK, Polkin RA, Ochkal SV, Stepanov IA, Makhambetov YT, Kerimbayev TT, Staren M, Belykh E, Preul MC (2018) Microvascular Anastomosis Training in Neurosurgery: A Review. Minimally invasive surgery 2018:6130286

7. Cooke M, Chaboyer W, Schluter P, Hiratos M (2005) The effect of music on preoperative anxiety in day surgery. Journal of advanced nursing 52:47-55

8. Eivazi S, Hafez A, FuhI W, Afkari H, Kasneci E, Lehecka M, Bednarik R (2017) Optimal eye movement strategies: a comparison of neurosurgeons gaze patterns when using a surgical microscope. Acta neurochirurgica 159:959-966

9. Fox KC, Nijeboer S, Dixon ML, Floman JL, Ellamil M, Rumak SP, Sedlmeier P, Christoff K (2014) Is meditation associated with altered brain structure? A systematic review and meta-analysis of morphometric neuroimaging in meditation practitioners. Neuroscience and biobehavioral reviews 43:48-73 10. Gasenzer ER, Kanat A, Neugebauer E (2017) Neurosurgery and Music; Effect of Wolfgang Amadeus Mozart. World neurosurgery 102:313-319

11. Hafez A, Huhtakangas J, Muhammad S, Lawton MT, Tanikawa R, Niemela M (2018) The Identification of Factors That Influence the Quality of Bypass Anastomosis and an Evaluation of the Usefulness of an Experimental Practical Scale in This Regard. World neurosurgery. doi: 10.1016/j.wneu.2018.09.031

12. Hafez A, Raj R, Lawton MT, Niemela M (2017) Simple training tricks for mastering and taming bypass procedures in neurosurgery. Surgical neurology international 8:295

13. Hetzler D (2011) Re: "Operating room sound level hazards for patients and physicians". Otology \& neurotology : official publication of the American Otological Society, American Neurotology Society [and] European Academy of Otology and Neurotology 32:345; author reply 345 14. Huotarinen A, Niemela M, Hafez A (2018) The impact of neurosurgical procedure on cognitive resources: Results of bypass training. Surgical neurology international 9:71

15. Katz JD (2014) Noise in the operating room. Anesthesiology 121:894-898

16. Kim DH, Dacey RG, Zipfel GJ, Berger MS, McDermott M, Barbaro NM, Shapiro SA, Solomon RA, Harbaugh R, Day AL (2017) Neurosurgical Education in a Changing Healthcare and Regulatory Environment: A Consensus Statement from 6 Programs. Neurosurgery 80:S75-S82 17. Klimo P, Jr., DeCuypere M, Ragel BT, McCartney S, Couldwell WT, Boop FA (2013) Career satisfaction and burnout among U.S. neurosurgeons: a feasibility and pilot study. World neurosurgery 80:e59-68

18. Kracht JM, Busch-Vishniac IJ, West JE (2007) Noise in the operating rooms of Johns Hopkins Hospital. The Journal of the Acoustical Society of America 121:2673-2680

19. Lee DJ, Kulubya E, Goldin P, Goodarzi A, Girgis F (2018) Review of the Neural Oscillations Underlying Meditation. Frontiers in neuroscience 12:178

20. Lippelt DP, Hommel B, Colzato LS (2014) Focused attention, open monitoring and loving kindness meditation: effects on attention, conflict monitoring, and creativity - A review. Frontiers in psychology 5:1083 

therapy, and Zen meditation for depression, anxiety, pain, and psychological distress. Journal of psychiatric practice 18:233-252

22. McConville J, McAleer R, Hahne A (2017) Mindfulness Training for Health Profession Students-The Effect of Mindfulness Training on Psychological Well-Being, Learning and Clinical Performance of Health Professional Students: A Systematic Review of Randomized and Non-randomized Controlled Trials. Explore 13:26-45

23. Moraes MM, Rabelo PCR, Pinto VA, Pires W, Wanner SP, Szawka RE, Soares DD (2018) Auditory stimulation by exposure to melodic music increases dopamine and serotonin activities in rat forebrain areas linked to reward and motor control. Neuroscience letters 673:73-78

24. Naranjo JR, Schmidt S (2012) Is it me or not me? Modulation of perceptual-motor awareness and visuomotor performance by mindfulness meditation. BMC neuroscience 13:88

25. Patel AD, Iversen JR, Chen Y, Repp BH (2005) The influence of metricality and modality on synchronization with a beat. Experimental brain research 163:226-238

26. Perlovsky L, Cabanac A, Bonniot-Cabanac MC, Cabanac M (2013) Mozart effect, cognitive dissonance, and the pleasure of music. Behavioural brain research 244:9-14

27. Repp BH, Penel A (2004) Rhythmic movement is attracted more strongly to auditory than to visual rhythms. Psychological research 68:252-270

28. Salimpoor VN, Benovoy M, Larcher K, Dagher A, Zatorre RJ (2011) Anatomically distinct dopamine release during anticipation and experience of peak emotion to music. Nature neuroscience $14: 257-262$

29. Salimpoor VN, Benovoy M, Longo G, Cooperstock JR, Zatorre RJ (2009) The rewarding aspects of music listening are related to degree of emotional arousal. PloS one 4:e7487

30. Shambo L, Umadhay T, Pedoto A (2015) Music in the operating room: is it a safety hazard? AANA journal 83:43-48

31. Simkin DR, Black NB (2014) Meditation and mindfulness in clinical practice. Child and adolescent psychiatric clinics of North America 23:487-534

32. Tang YY, Tang R, Posner MI (2016) Mindfulness meditation improves emotion regulation and reduces drug abuse. Drug and alcohol dependence 163 Suppl 1:S13-18

33. Tuladhar R, Bohara G, Grigolini P, West BJ (2018) Meditation-Induced Coherence and Crucial Events. Frontiers in physiology 9:626

34. Weible AP, Piscopo DM, Rothbart MK, Posner MI, Niell CM (2017) Rhythmic brain stimulation reduces anxiety-related behavior in a mouse model based on meditation training. Proceedings of the National Academy of Sciences of the United States of America 114:2532-2537

35. Wilkins RW, Hodges DA, Laurienti PJ, Steen M, Burdette JH (2014) Network science and the effects of music preference on functional brain connectivity: from Beethoven to Eminem. Scientific reports 4:6130

36. Yadav YR, Parihar V, Ratre S, Kher Y, Iqbal M (2016) Microneurosurgical Skills Training. Journal of neurological surgery. Part A, Central European neurosurgery 77:146-154

37. Zatorre RJ, Chen JL, Penhune VB (2007) When the brain plays music: auditory-motor interactions in music perception and production. Nature reviews. Neuroscience 8:547-558 